PROCEEDINGS OF THE

AMERICAN MATHEMATICAL SOCIETY

Volume 129, Number 2, Pages 567-572

S 0002-9939(00)06048-2

Article electronically published on October 10, 2000

\title{
A NOTE ON NON-UNIVALENT HARMONIC MAPS BETWEEN SURFACES
}

\author{
TOM Y. H. WAN
}

(Communicated by Peter Li)

\begin{abstract}
We show that a decomposition theorem of Duren-Hengartner about planar harmonic maps can be generalized to give a necessary and sufficient condition for a harmonic map between smooth surfaces to be decomposable as a holomorphic map followed by a univalent harmonic embedding.
\end{abstract}

\section{INTRODUCTION}

Due to its application in Reimann surfaces theory, the study of harmonic diffeomorphisms between surfaces has attracted a lot of attention. The class of harmonic diffeomorphisms seems to be a good extension of the class of univalent functions in complex function theory. So it is natural to consider the class of non-univalent harmonic maps and hopefully it is also a good extension of the class of holomorphic covering maps.

It is well known that, for a harmonic map $f: M \rightarrow N$ from a Riemann surface $M$ to a manifold $N$ and a holomorphic (anti-holomorphic) map $\varphi: M^{\prime} \rightarrow M$ from another Riemann surface $M^{\prime}$ to $M$, the composition $f \circ \varphi: M^{\prime} \rightarrow N$ is also a harmonic map. This observation provides many examples of branched harmonic covering maps as long as we have a univalent harmonic map to start with. Therefore it is interesting to know when a non-univalent harmonic map is decomposable in this form.

This problem was studied by Duren-Hengartner [2] for the case of planar harmonic maps, i.e. harmonic maps from domains in $\mathbb{C}$ to $\mathbb{C}$. They found a necessary and sufficient condition for the existence of such a decomposition. In this note, we show that this result can be generalized to the non-linear situation of harmonic maps between surfaces.

The main result in this note was done while the author visited Tohoku University under the JSPS fellowship program in 1997. The author would like to thank S. Nishikawa for applying the fellowship and for all his help while the author stayed in Sendai, Japan. The author would also like to thank T. Au and L. F. Tam for their interest in this work and helpful discussions.

Received by the editors May 22, 1997.

2000 Mathematics Subject Classification. Primary 58E20.

This research is partially supported by the Earmarked Grant Hong Kong and JSPS fellowship.

(C)2000 American Mathematical Society 


\section{MAIN THEOREM}

Let us first point out an obvious necessary condition. If $f$ is an orientation preserving (reversing) harmonic diffeomorphism and $\varphi$ is holomorphic (anti-holomorphic), then the Jacobian of $f \circ \varphi$ does not change sign, i.e. $J(f \circ \varphi) \geq 0$ or $\leq 0$. Therefore, only harmonic maps with non-negative or non-positive Jacobian are decomposable as we want. Moreover, it is enough to consider only those harmonic maps with non-negative Jacobian since we can always compose a harmonic map with a complex conjugate to change the sign of its Jacobian and remain harmonic. Now, let us state our main theorem.

Theorem 1.1 (Main Theorem). Suppose $M$ is a Riemann surface, $N$ is a Riemannian surface, and $u: M \rightarrow N$ is a non-constant harmonic map. If $J(u) \geq 0$ on $M, J(u)>0$ somewhere, and

$$
\frac{\bar{u}_{z}}{u_{z}}\left(z_{1}\right)=\frac{\bar{u}_{z}}{u_{z}}\left(z_{2}\right)
$$

whenever $u\left(z_{1}\right)=u\left(z_{2}\right)$ and $J(u)\left(z_{i}\right)>0(i=1,2)$, then there exist a Riemann surface $\Sigma$ homeomorphic to $u(M)$, a holomorphic mapping $\varphi: M \rightarrow \Sigma$, and a harmonic embedding $v: \Sigma \rightarrow N$ such that $u=v \circ \varphi$.

Moreover, the converse is also true.

Remark 1.1. Our condition looks a little bit different from that in 2 . However, they are in fact equivalent by the result of Schoen-Yau [5] that $\left|\bar{u}_{z} / u_{z}\right| \neq 1$ follows from $J(u) \geq 0($ or $\leq 0)$ unless $J(u) \equiv 0$.

Proof. The converse is easy to prove. We already pointed out that $J(u) \geq 0$. If $J(u) \equiv 0$, then $\varphi^{\prime} \equiv 0$, which is impossible since $u$ is not a constant mapping. Now by direct calculation, we find

$$
\bar{u}_{z}=\left(\bar{v}_{w} \circ \varphi\right) \varphi^{\prime} \quad \text { and } \quad u_{z}=\left(v_{w} \circ \varphi\right) \varphi^{\prime} .
$$

Since $v$ is one-to-one, the equality $u\left(z_{1}\right)=u\left(z_{2}\right)$ implies $\varphi\left(z_{1}\right)=\varphi\left(z_{2}\right)$. Moreover, $J(u)\left(z_{i}\right)>0$ implies $\varphi^{\prime}\left(z_{i}\right) \neq 0$ for $i=1,2$. Therefore

$$
\begin{aligned}
\frac{\bar{u}_{z}}{u_{z}}\left(z_{1}\right) & =\frac{\bar{v}_{w}\left(\varphi\left(z_{1}\right)\right) \varphi^{\prime}\left(z_{1}\right)}{v_{w}\left(\varphi\left(z_{1}\right)\right) \varphi^{\prime}\left(z_{1}\right)}=\frac{\bar{v}_{w}\left(\varphi\left(z_{1}\right)\right)}{v_{w}\left(\varphi\left(z_{1}\right)\right)} \\
& =\frac{\bar{v}_{w}\left(\varphi\left(z_{2}\right)\right)}{v_{w}\left(\varphi\left(z_{2}\right)\right)}=\frac{\bar{v}_{w}\left(\varphi\left(z_{2}\right)\right) \varphi^{\prime}\left(z_{2}\right)}{v_{w}\left(\varphi\left(z_{2}\right)\right) \varphi^{\prime}\left(z_{2}\right)} \\
& =\frac{\bar{u}_{z}}{u_{z}}\left(z_{2}\right) .
\end{aligned}
$$

Now suppose $u$ satisfies the condition that $J(u) \geq 0$ on $M, J(u)>0$ somewhere, and (1.1). In order to construct the Riemann surface $\Sigma$ and the holomorphic map $\varphi$, we will define a new complex structure $\tau$ on the image $\Omega=u(M)$ such that $u: M \rightarrow \Omega$ is holomorphic with respect to $\tau$. We first note from the result of Schoen-Yau [5 that the critical points of $u$ are isolated since $J(u) \geq 0$ and $J(u) \not \equiv 0$. Let $\mathcal{C}=\{p \in N$ : all preimages of $p$ are critical $\} \subset N$. Then $\mathcal{C}$ is a subset of the set of all critical values and consists of isolated points in $u(M)$ if it is non-empty. Otherwise, there exists a sequence $\left\{p_{j}\right\} \in \mathcal{C}$ and a point $p \in u(M)$ such that $p_{j} \rightarrow p$. If $p \notin \mathcal{C}$, then there exists a point $z \in u^{-1}(p)$ such that $u$ is locally diffeomorphic near $z$. So we can find preimages $z_{j}$ of $p_{j}$ approaching $z$ for large $j$. Since $p_{j} \in \mathcal{C}, z_{j}$ are critical points of $u$. Hence, by the smoothness of $u$, $z$ must also be a critical point of $u$, which contradicts the choice of $z$. Therefore, 
we have $p \in \mathcal{C}$. However, we can apply a similar argument to show that this is also impossible. In fact, let $z$ be a preimage of $p$. Then $z$ must be critical and, by the result of Schoen-Yau, $u$ behaves like a branched covering near $z$. So $u$ maps a neighborhood of $z$ onto a neighborhood of $p$. Therefore we have again a sequence of critical points $z_{j}$ approaching $z$, which is a contradiction.

Now we define a new complex structure $\mathcal{A}$ on $\Omega \backslash \mathcal{C}$ such that $u: M \backslash u^{-1}(\mathcal{C}) \rightarrow$ $\Omega \backslash \mathcal{C}$ is holomorphic with respect to $\mathcal{A}$ and then show that $\mathcal{A}$ can be extended to a complex structure $\tau$ on $\Omega$. The obvious choice for $\mathcal{A}$ is

$$
\mathcal{A}=\left\{\left(u(O), \phi \circ\left(\left.u\right|_{O}\right)^{-1}\right)\right\}
$$

where each $O$ is an open set contained in some coordinate chart with coordinate function $\phi$ of $M$ such that $\left.u\right|_{O}: O \rightarrow u(O)$ is a diffeomorphism. To show that $\mathcal{A}$ defines a complex structure, we take two arbitrary elements $\left(u\left(O_{1}\right), \phi_{1} \circ\left(\left.u\right|_{O_{1}}\right)^{-1}\right)$ and $\left(u\left(O_{2}\right), \phi_{2} \circ\left(\left.u\right|_{O_{2}}\right)^{-1}\right)$ in $\mathcal{A}$ such that $V=u\left(O_{1}\right) \cap u\left(O_{2}\right)$ is non-empty. Then the transition function $\left(\phi_{2} \circ\left(\left.u\right|_{O_{2}}\right)^{-1}\right) \circ\left(\phi_{1} \circ\left(\left.u\right|_{O_{1}}\right)^{-1}\right)^{-1}$ is holomorphic if and only if $f=\left.\left(\left.u\right|_{O_{2}}\right)^{-1} \circ u\right|_{O_{1}}$ is holomorphic on $\left(\left.u\right|_{O_{1}}\right)^{-1}(V)$. To check this, we take any $z \in\left(\left.u\right|_{O_{1}}\right)^{-1}(V)$. Then $u(z)=u(f(z)), J(u)(z)>0$, and $J(u)(f(z))>0$. By condition (1.1),

$$
\frac{\bar{u}_{z}}{u_{z}}(f(z))=\frac{\bar{u}_{z}}{u_{z}}(z)=\frac{\overline{u_{z}}(f(z)) \bar{f}_{z}+\bar{u}_{z}(f(z)) f_{z}}{u_{z}(f(z)) f_{z}+u_{\bar{z}}(f(z)) \bar{f}_{z}} .
$$

This is equivalent to

$$
\left(\left|u_{z}\right|^{2}(f(z))-\left|u_{\bar{z}}\right|^{2}(f(z))\right) \bar{f}_{z}(z)=0 .
$$

Using again $J(u)(f(z))>0$ for all $z \in V_{j}$, we see that $f$ has to be holomorphic. So $\mathcal{A}$ defines a complex structure on the set $\bigcup u(O)=\Omega \backslash \mathcal{C}$.

To prove that $\mathcal{A}$ extends to the whole $\Omega$, we recall that $\mathcal{C}$ consists of isolated points in $\Omega$. Hence for any $p \in \mathcal{C}$, there exists a neighborhood $U$ of $p$ such that $(U \backslash\{p\}) \cap \mathcal{C}=\emptyset$, i.e. $U \backslash\{p\} \subset \Omega \backslash \mathcal{C}$. The new complex structure $\mathcal{A}$ on $\Omega \backslash \mathcal{C}$ induces a complex structure on $U \backslash\{p\}$. According to the uniformization theorem, $U \backslash\{p\}$ is conformally equivalent to either $D_{1} \backslash\{0\}$ or $D_{1} \backslash D_{r}$ for some $r \in(0,1)$, where $D_{r}=\{|z|<r\}$. We want to show that the latter case is impossible. To see this, let $z$ be a critical point of $u$ such that $u(z)=p$. Then there exists a neighborhood $V$ of $z$ such that $u(V) \subset U$. With respect to the complex structure $\mathcal{A}, u: V \backslash\{z\} \rightarrow U \backslash\{p\}$ is holomorphic. Hence, the removable singularity theorem implies that $u$ extends across $z$. So $U \backslash\{p\}$ is conformally equivalent to $D_{1} \backslash\{0\}$. Thus we can extend the complex structure $\mathcal{A}$ on $U \backslash\{p\}$ to $U$. In this way, we see that the complex structure $\mathcal{A}$ defined on $\Omega \backslash u(\mathcal{C})$ extends to a complex structure $\tau$ on $\Omega$. Using the removable singularity theorem again, we conclude that $u: M \rightarrow \Omega$ is holomorphic with respect to $\tau$.

Finally, denote the Reimann surface given by $\Omega$ with complex structure $\tau$ by $\Sigma$ and the holomorphic map $u: M \rightarrow \Sigma$ by $\varphi$. Then $u=v \circ \varphi$ with $v=\operatorname{Id}_{\Omega}$ the identity map on $\Omega$. Using the conformal invariance of harmonic maps from surfaces, it is easy to see that $v$ is a harmonic embedding when considered as a map from the Riemann surface $\Sigma$ into the Riemannian surface $N$. This completes the proof of the theorem.

Since holomorphic maps are branched coverings, we have

Corollary 1.2. Let $u: M \rightarrow N$ be a harmonic map between surfaces satisfying the condition in the main theorem. Then $u$ is a branched covering. 


\section{MAPS FROM THE COMPLEX PLANE}

From the main theorem, we see that the result of Duren-Hengartner can be extended to harmonic maps between arbitrary surfaces. However, the main theorem is weaker than their result in the sense that it does not provide enough information about the Riemann surface $\Sigma$. The theorem in [2] shows that $\Sigma$ is in fact a domain in the complex plane $\mathbb{C}$ when both $M$ and $N$ are domains in $\mathbb{C}$. In this section, we show a similar result for the case that the domain surface $M$ is the entire complex plane $\mathbb{C}$ and $N$ is a complete simply-connected surface with non-positive curvature.

Theorem 2.1. Suppose $u: \mathbb{C} \rightarrow N$ is a non-constant harmonic map from the complex plane into a complete simply-connected surface with non-positive curvature. If $J(u) \geq 0$ on $\mathbb{C}$ and $J(u)>0$ somewhere, and if

$$
\frac{\bar{u}_{z}}{u_{z}}\left(z_{1}\right)=\frac{\bar{u}_{z}}{u_{z}}\left(z_{2}\right)
$$

whenever $u\left(z_{1}\right)=u\left(z_{2}\right)$ and $J(u)\left(z_{i}\right)>0(i=1,2)$, then there is a holomorphic function $\varphi: \mathbb{C} \rightarrow \mathbb{C}$ and a harmonic embedding $v: \mathbb{C} \rightarrow N$ such that $u=v \circ \varphi$.

Proof. By the main theorem, there is a Riemann surface $\Sigma$, a holomorphic map $\hat{\varphi}: \mathbb{C} \rightarrow \Sigma$, and a harmonic embedding $\hat{v}: \Sigma \rightarrow N$ such that $u=\hat{v} \circ \hat{\varphi}$. Since $\mathbb{C}$ is simply-connected, $\hat{\varphi}$ lifts to a holomorphic mapping from $\mathbb{C}$ to the universal covering of $\Sigma$. Then the Liouville theorem implies that the universal covering of $\Sigma$ is conformally equivalent to $\mathbb{C}$. Therefore, $\Sigma$ is conformally equivalent to either $\mathbb{C}$ or $\mathbb{C} \backslash\{0\}$.

If $\Sigma$ is conformally equivalent to $\mathbb{C}$ and $\mathcal{R}: \Sigma \rightarrow \mathbb{C}$ is a conformal equivalence, then $\varphi=\mathcal{R} \circ \hat{\varphi}$ is a holomorphic function and $\hat{v} \circ \mathcal{R}^{-1}: \mathbb{C} \rightarrow N$ is a harmonic embedding such that $u=v \circ \varphi$. So we are done in this case.

If $\Sigma$ is conformally equivalent to $\mathbb{C} \backslash\{0\}$, then we have a holomorphic function $\varphi$ whose image omits the point 0 and a harmonic embedding $v$ from $\mathbb{C} \backslash\{0\}$ to $N$ such that $u=v \circ \varphi$. The question in this case is whether $v$ extends across 0 (or $\infty$ ) to a harmonic embedding on the whole $\mathbb{C}$.

Since $v$ is an embedding, its image has two boundary components, one corresponds to the origin and the other corresponds to infinity. By the simplyconnectedness of $N$, one of the boundary components must be bounded. Therefore, we may assume that $v(z)$ is bounded near the origin (otherwise, we consider $\tilde{v}(z)=v(1 / z))$, say bounded on $D_{1} \backslash\{0\}$. Define a harmonic map $f(z)=v\left(e^{z}\right)$ on the left half-plane $\{\operatorname{Re}(z)<0\}$. For any point $z=-\sigma+i$ with $\sigma>0$, the disc $D_{\sigma}(z)$ with radius $\sigma$ centered at $z$ is contained in the left half-plane. Since the image of $v$, hence the image of $f$, is bounded and $N$ has non-positive curvature, the gradient estimate of Cheng [1] implies that there is a constant $C>0$, independent of $z$, such that

$$
e(f)(z) \leq \frac{C}{\sigma^{2}}
$$

where $e(f)$ is the energy density of $f$. Now the holomorphic map $e^{z}$ maps $\{z=$ $-\sigma+i t \mid \sigma>0,0<t<2 \pi\}$ univalently onto $D_{1} \backslash(-1,0)$. Therefore, by conformal 
invariance of the two-dimensional energy functional and the gradient estimate,

$$
\begin{aligned}
\int_{D_{1} \backslash\{0\}} e(v) & =\int_{D_{1} \backslash(-1,0)} e(v) \\
& =\int_{\{z=-\sigma+i t \mid \sigma>0,0<t<2 \pi\}} e(f) \\
& <+\infty .
\end{aligned}
$$

By the result of Sacks-Uhlenbeck [3], $v$ extends to a smooth harmonic map across the origin. This completes the proof of the theorem.

Corollary 2.2. Let $u: \mathbb{C} \rightarrow N$ be a non-constant harmonic map from the complex plane into a complete simply-connected surface with non-positive curvature satisfying the condition in the main theorem. Then the image of $u$ is a simply-connected subdomain in $N$ or a simply-connected subdomain with a point deleted.

Proof. Use Theorem 2.1 and Picard's theorem.

\section{UNIQUENESS}

Finally we would like to make two observations about uniqueness. The first one is the uniqueness of the representation given in the main theorem. This is exactly the same result given in [2].

Proposition 3.1. Suppose that a harmonic map $u: M \rightarrow N$ between surfaces $M$ and $N$ can be decomposed as $u=v_{1} \circ \varphi_{1}$ and $u=v_{2} \circ \varphi_{2}$, where $\varphi_{i}$ are holomorphic maps from $M$ to some Reimann surfaces $\Sigma_{i}$, and $v_{i}: \Sigma_{i} \rightarrow N$ are harmonic embeddings. Then there is a conformal map $\xi$ from $\varphi_{1}(M)$ onto $\varphi_{2}(M)$ such that

$$
v_{2}=v_{1} \circ \xi^{-1}, \quad \varphi_{2}=\xi \circ \varphi_{1} .
$$

Proof. One checks easily that $\xi$ defined by $v_{2}^{-1} \circ v_{1}$ is conformal.

The second observation is about entire planar harmonic maps, i.e. harmonic maps from the entire complex plane to itself.

Proposition 3.2. Any orientation preserving (or reversing) entire planar harmonic map which does not map into a geodesic is a composition of a real linear transformation and a holomorphic function.

Proof. A simple argument in [4] used to prove that harmonic diffeomorphisms of $\mathbb{C}$ onto itself is a real linear transformation works for the proposition. What we want to point out here is that this proposition is in fact a special case of our theorems. For a planar harmonic map $u$, say orientation preserving, the dilatation function $\bar{u}_{z} / u_{z}$ is holomorphic. So if it is defined on the entire complex plane, it must be a constant $a$ with $|a|<1$. Therefore, the conditions in our theorems are satisfied trivially. Hence $u$ is decomposable as a composition of a harmonic embedding from $\mathbb{C}$ to itself, which has to be a real linear transformation, and a holomorphic function.

Unlike the entire plane $\mathbb{C}$, there is an example of an orientation preserving planar harmonic map defined on the unit disk which does not satisfy the condition (1.1) [2]. It seems an interesting problem to find a condition on the surfaces such that a corresponding result of Proposition 3.2 holds. For example, is it true that any nonunivalent orientation preserving harmonic map from the entire complex plane $\mathbb{C}$ to 
a complete simply-connected surface with non-positive curvature is decomposable as a holomorphic function followed by a univalent harmonic map?

\section{REFERENCES}

[1] S. Y. Cheng, Liouville theorem for harmonic maps, Proceedings of Symposia in Pure Mathematics, 36 (1980) 147-151. MR 81i:58021

[2] P. Duren \& W. Hengartner, A decomposition theorem for planar harmonic mappings, Proc. AMS 124 no. 4 (1996) 1191-1195. MR 96g:31001

[3] J. Sacks \& K. Uhlenbeck, The existence of minimal immersions of 2-sphere, Ann. of Math. 113 (1981) 1-24. MR 82f:58035

[4] R. Schoen, The role of harmonic mappings in rigidity and deformation problems, Complex Geometry (Osaka, 1990), Lecture Notes in Pure \& Applied Mathematics, 143, Dekker, New York, 1993, 179-200. MR 94g:58055

[5] R. Schoen \& S. T. Yau, Lectures on harmonic maps, International Press, 1997. MR 98i:58072

Department of Mathematics, The Chinese University of Hong Kong, Shatin, N.T., HONG KONG

E-mail address: tomwan@math.cuhk.edu.hk 